\title{
A nonlinear parametric model based on a power law relationship for predicting the coastal tsunami height
}

\author{
Masashi Yoshikawa ${ }^{1} \cdot$ Yasuhiko Igarashi $^{2} \cdot$ Shin Murata ${ }^{1} \cdot$ Toshitaka Baba $^{3} \cdot$ Takane Hori $^{4} \cdot$ Masato Okada $^{1}$
}

Received: 7 March 2018 / Accepted: 25 November 2018 / Published online: 10 June 2019

(c) The Author(s) 2019

\begin{abstract}
When a subduction-zone earthquake occurs, the tsunami height must be predicted to cope with the damage generated by the tsunami. Therefore, tsunami height prediction methods have been studied using simulation data acquired by large-scale calculations. In this research, we consider the existence of a nonlinear power law relationship between the water pressure gauge data observed by the Dense Oceanfloor Network System for Earthquakes and Tsunamis (DONET) and the coastal tsunami height. Using this relationship, we propose a nonlinear parametric model and conduct a prediction experiment to compare the accuracy of the proposed method with those of previous methods and implement particular improvements to the extrapolation accuracy.
\end{abstract}

Keywords Nonlinear parametric model $\cdot$ Tsunami height prediction $\cdot$ Power law $\cdot$ Extrapolation

\section{Introduction}

Tsunami early warning systems using water pressure gauges and global positioning system wave gauges operate around the world to cope with damages caused by tsunami waves. Accordingly, real-time water pressure gauge data are widely used for tsunami early warning systems. For example, in Japan, the Dense Oceanfloor Network System for Earthquakes and Tsunamis (DONET), which enables the acquisition of submarine data in real time, was constructed in the Nankai Trough (Kaneda et al. 2015).

Many real-time tsunami prediction studies used water pressure gauge data from the floor. For instance, Tsushima

Masato Okada

okada@k.u-tokyo.ac.jp

1 Department of Complexity Science and Engineering, The University of Tokyo, 5-1-5, Kashiwanoha, Kashiwa, Chiba 277-8561, Japan

2 Japan Science and Technology Agency, PRESTO, 4-1-8 Honcho, Kawaguchi, Saitama 332-0012, Japan

3 Graduate School of Science and Technology, Tokushima University, 2-24, Shinkura-cho, Tokushima 770-8501, Japan

4 Research and Development Center for Earthquake and Tsunami, Japan Agency for Marine-Earth Science and Technology (JAMSTEC), 3173-25, Showa-machi, Kanazawaku, Yokohama, Kanagawa 236-0001, Japan et al. $(2009,2011)$ proposed a prediction method that combines water pressure gauge data with the estimation results of a tsunami source and estimated the initial distribution of the sea surface around the tsunami source. In addition, they accurately predicted a tsunami waveform within 20 minutes after the Tohoku Earthquake (Tsushima et al. 2009, 2011). However, a tsunami produced by an earthquake in the Nankai Trough arrives after only a few minutes, and thus, a more rapid tsunami height prediction scheme is needed. To perform such a rapid prediction, it is considered more effective to forecast the tsunami height from only water pressure gauge data (Baba et al. 2004, 2014; Hayashi 2010) because this approach does not need an estimation of the initial distribution of the sea surface and can predict the height with a smaller amount of computational resources.

Therefore, instead of using a conventional forward calculation strategy that predicts the tsunami height after estimating the earthquake parameters, such as the earthquake magnitude (Fig. 1a) (Tsushima et al. 2009, 2011), we decided to use a strategy that predicts the tsunami height directly from water pressure gauge data (Fig. 1b) (Baba et al. 2014; Igarashi et al. 2016).

To construct a tsunami prediction model using only water pressure gauge data, we simulated the water levels at the ocean floor and on the coast by assuming various parameters, including the fault model and seismic source. Then, we constructed a database based on these assumed parameters 
Fig. 1 Schematic diagrams of two strategies employed to predict the tsunami height showing how to predict the tsunami height in real time using an earthquake parameter $\theta$ and the data obtained by simulation $s$ and $d$
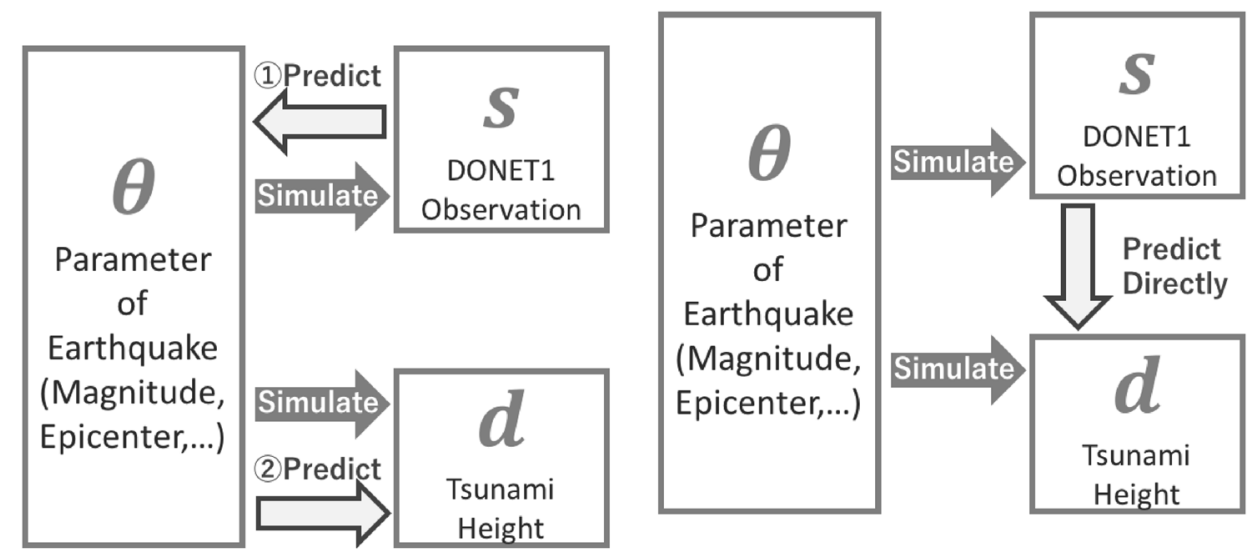

(a) Conventional forward calculation strategy (b) Tsunami height prediction strategy using the rela-

tionship between data and regressed the tsunami height at the coast from the water pressure gauge data (Baba et al. 2014; Igarashi et al. 2016). Two methods, namely, one using linear regression (Baba et al. 2014) and one using Gaussian process (GP) regression (Igarashi et al. 2016), have been proposed to regress the tsunami height from the water pressure gauge data.

Regression models are classified as either parametric model or nonparametric models. In a parametric model, the relationship between the data is modeled by supposing that the relationship can be expressed by a parameterized formula. Linear regression is classified as a parametric mode in which linearity is supposed. Unfortunately, the prediction method using linear regression exhibits a low accuracy because the relationship is actually nonlinear (Baba et al. 2014). In contrast, in a nonparametric model, strong assumptions are not placed on the relationship; as a result, flexible modeling can be performed. GP regression is classified as a nonparametric model. Because the modeling scheme is strongly affected by the data, the prediction method using GP regression is unable to provide effective prediction beyond the range of training data, and it also demonstrates a low extrapolation accuracy. Therefore, to construct a model with a high accuracy and a high extrapolation accuracy (Igarashi et al. 2016), it is necessary to have additional information regarding the relationship between the data, and a parametric model should be constructed under reasonable assumptions.

In this study, we plot observed water pressure gauge data and the coastal tsunami heights in a log-log graph, in which the data appear to be linearly distributed; consequently, we believe that a power law relationship exists between the gauge data and tsunami height. Accordingly, we propose a nonlinear parametric model to predict the tsunami height on the basis of a power law, that is, as the weighted sum of an exponentiated observed value.

To evaluate the prediction performance of our proposed method, we calculate the prediction performance in two ways. First, we assume that the test data are drawn from the same population as the training data, and we investigate the prediction performance under expected circumstances following a previous study (Igarashi et al. 2016). However, in practice, we cannot deny the possibility that the coastal tsunami height will exceed the scope of the assumption; hence, we also evaluate the prediction performance under unexpected circumstances through extrapolation and compare the extrapolation accuracies of the previous methods (Baba et al. 2014; Igarashi et al. 2016) with that of our proposed nonlinear parametric model. In this investigation, we target Owase on the east coast of the Kii Peninsula, Japan.

\section{Simulation and dataset}

\section{Simulation}

We built a database to construct numerous fault models for predicting the height of a tsunami arriving at the Kii Peninsula. We prepared 1506 fault models by considering the surface shape of the Philippine Sea Plate (Baba et al. 2002) and the source processes related to the 1944 Tokai Earthquake and the 1946 Nankai Earthquake (Kanamori 1972; Baba and Cummins 2005). Figure 2 shows the positions of the fault models used in this study with rectangles (Fig. 2). We set the shallowest depth of the faults to range from 5 to $25 \mathrm{~km}$, the dip to range from $5^{\circ}$ to $25^{\circ}$, and the magnitude to range from 7.2 to 8.4. The fault length, width, and amount of slip were determined according to a scaling law (Utsu 2001). The strike and the rake were set at a constant values of $240^{\circ}$ and $90^{\circ}$, respectively. For the sake of simplicity, a uniform fault was assumed in each of the 1506 fault models.

To simulate a tsunami from the fault models, we used the tsunami simulation code JAGURS (Baba et al. 2016). We simulated the tsunami propagation over a period 


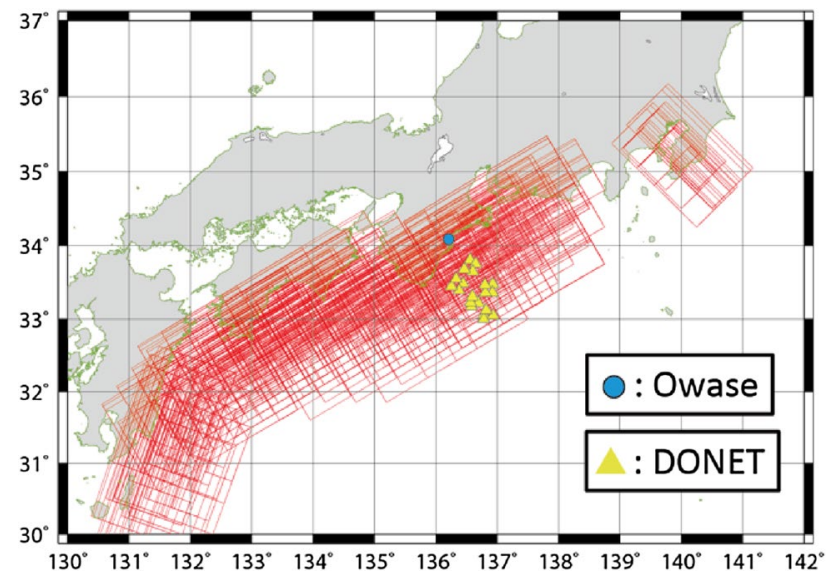

Fig. 2 Positions of the fault models and observation points used in this study. We prepared 1506 fault models (rectangles). This figure is adapted from Igarashi et al. (2016), Fig. 1a and shows the location of DONET1 observation points (triangles) and Owase (circle)

of $3 \mathrm{~h}$ following the occurrence of an earthquake and recorded the waveforms every second for each fault model $(T=3 \mathrm{~h} \times 3600 \mathrm{~s} / \mathrm{h}=10,800 \mathrm{~s})$.

\section{Dataset}

Through this simulation, we obtained the change in the water level $\eta$, which obeys the equation of motion in nonlinear long wave theory, and the water depth $H$, which varies as a result of crustal deformation. We then defined the deviation from the mean sea level as the tsunami height in this paper and set the maximum value of the tsunami height at Owase in scenario $n(n=1, \ldots, 1506)$ as $d(n)$. Next, we converted the total water depth (water depth $H+$ water level change $\eta$ ) into the change in the water pressure changes and then defined the change in the water pressure change at the $i$ th $(i=1, \ldots, S)$ observation point of DONET1 as $p_{i}(n, t)(t=1, \ldots, T)$. Figure 3 shows the waveform measured at Owase and the water pressure data obtained by DONET1 for scenario $n=1057$ : the red line shows the tsunami height waveform at Owase, and the red point shows its maximum value $d$, while the light blue broken lines show each set of water pressure data observed by each DONET1 observation point, and the blue line shows the averaged waveform of the absolute water pressure data. Figure 3 shows the maximum value of average absolute value $s(n)$ (blue point), which exceeds $400 \mathrm{hPa}$ and the maximum tsunami height $d(n)$ (red point). There is a strong positive correlation between observed water pressure gauge data and the coastal tsunami height in Owase (Baba et al. 2014), and thus, the coastal tsunami height in Owase is high in this scenario.

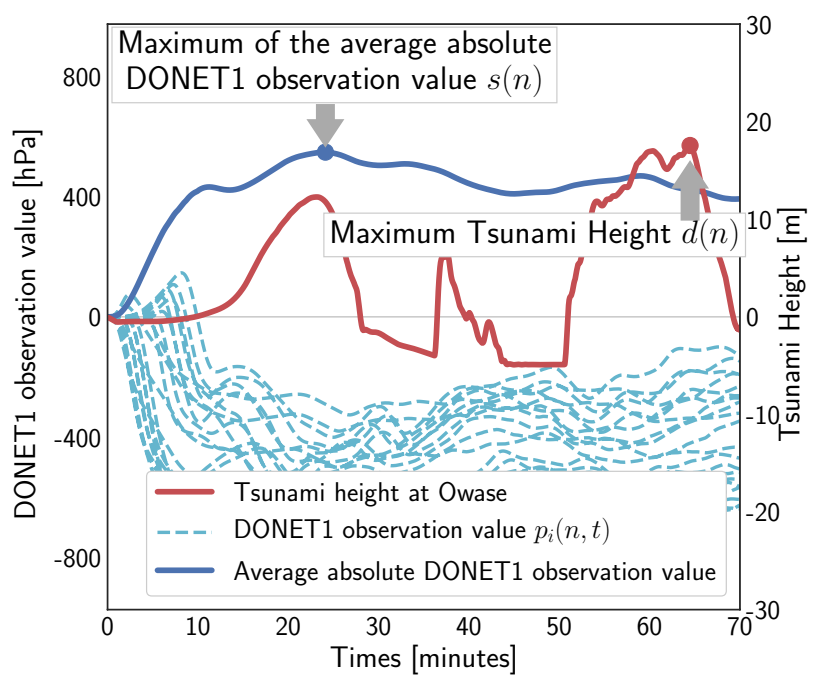

Fig. 3 Hydrostatic pressure changes at the DONET1 observation points $p_{i}(n, t)(i=1, \ldots, S=20)$ (blue dashed lines) and the tsunami height at Owase (red line). This figure also shows the average value of absolute hydrostatic pressure change (blue line) used in a previous study (Baba et al. 2014). In this figure, we show the maximum value of average absolute value $s(n)$ (blue point), which exceeds $400 \mathrm{hPa}$, and the maximum tsunami height $d(n)$ (red point)

\section{Method}

\section{Linear regression using the maximum mean (MM regression)}

A clear correlation was found between the average absolute DONET1 hydrostatic pressure and the maximum coastal tsunami height (Fig. 4a). On the basis of that relationship, a tsunami height prediction method using linear regression was proposed in previous work (Baba et al. 2014). We set $s(n)=\max _{t} \frac{1}{S} \sum_{i=1}^{S}\left|p_{i}(n, t)\right|$ as the maximum value of the average absolute value observed by the water pressure gauge during scenario $n$ and call it the MM. This prediction algorithm is then expressed as

$\hat{d}_{\mathrm{MM}}(n)=w_{\mathrm{MM}}^{1} s(n)+w_{\mathrm{MM}}^{0}$,

where $\hat{d}_{\mathrm{MM}}(n)$ is the predicted tsunami height in scenario $n$, and $w_{\mathrm{MM}}^{1}$ and $w_{\mathrm{MM}}^{0}$ are regression coefficients. $d(n)$ is fitted by linear regression, and the regression coefficients are determined by training data and the least squares method.

\section{Gaussian process (GP) regression}

Predicting the height of a tsunami by linear regression is less accurate because the relationship between $d(n)$ and $s(n)$ is actually nonlinear (Igarashi et al. 2016). To address this problem, a tsunami height prediction method using GP regression (Rasmussen and Williams 2006) was proposed in a previous study (Igarashi et al. 2016). GP regression 
Fig. 4 Relationship between $s(n)$ and $d(n)$. a The relationship is plotted linearly. b The relationship is plotted as a log$\log$ graph

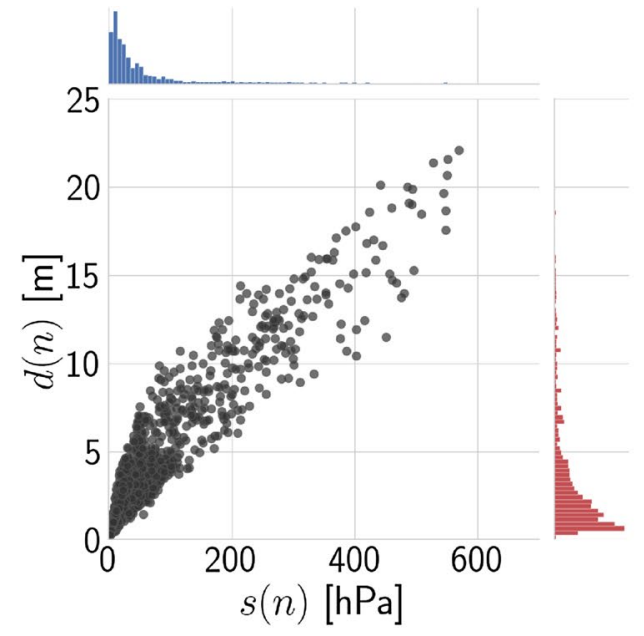

(a)

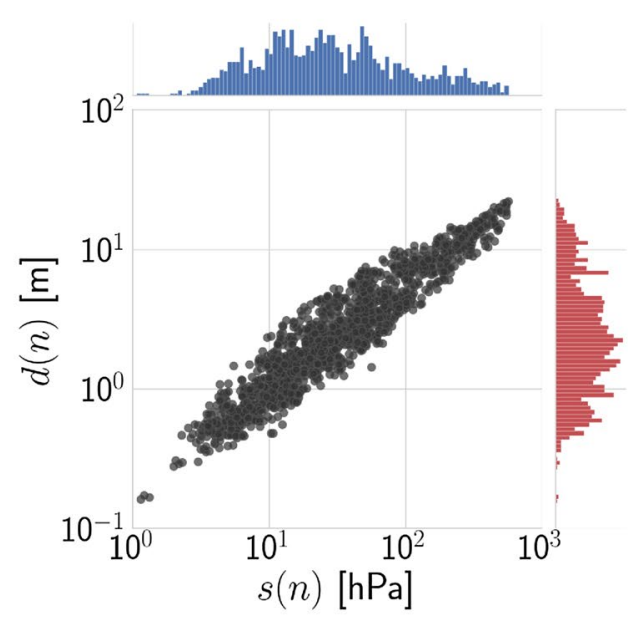

(b) can be applied to nonlinear data and is thus widely used in prediction and optimization fields (e.g., Kocijan et al. 2004; Krause et al. 2008). Accordingly, GP regression can be employed with a high accuracy to predict the height of a tsunami as the weighted sum of the kernel function of all training data. Moreover, the previously proposed prediction algorithm does not employ the mean value of all observed points; rather, the algorithm uses the individual values, that is, $s_{i}(n)=\max _{t}\left|p_{i}(n, t)\right|$ consisting of $S$ values.

Let us consider the formulation of GP regression concretely. We define a Gaussian kernel function constructed around training data (Igarashi et al. 2016) as follows:

$k(\mathbf{s}(n), \mathbf{s}(m))=\exp \left(-\beta|\mathbf{s}(n)-\mathbf{s}(m)|^{2}\right)$,

where $\mathbf{s}(n)=\left[s_{1}(n), \ldots, s_{S}(n)\right]$. The Gaussian kernel function has a high value when two inputs are similar, namely, when the Euclidean distance between $\mathbf{s}(n)$ and $\mathbf{s}(m)$ is small. The Gaussian kernel function corresponds to the covariance between the prior distributions of $d(n)$ and $d(m)$; this correlation can be interpreted insomuch that we can insert a prior knowledge, that is, the higher the value of the kernel function is, the higher the correlation between $d(n)$ and $d(m)$.

We define $\mathbf{s}(1), \ldots, \mathbf{s}\left(N_{T}\right)$ and $\mathbf{d}=\left[d(1), \ldots, d\left(N_{T}\right)\right]^{\top}$ as a training data set. We also define $\mathbf{s}_{\text {new }}$ and $d_{\text {new }}$ as a test data set.

We then introduce a matrix consisting of kernels denoted $\mathbf{K}$ between the training data. The $(i, j)$ th component of $\mathbf{K}$ is $k(\mathbf{s}(i), \mathbf{s}(j))$. We also introduce $\mathbf{K}$ as a vector consisting of kernels between the training data and the test data. The $i$ th component of $\mathbf{k}$ is $k\left(\mathbf{s}(i), \mathbf{s}_{\text {new }}\right)$, that is, $\mathbf{k}=\left[k\left(\mathbf{s}(1), \mathbf{s}_{\text {new }}\right), \ldots, k\left(\mathbf{s}(i), \mathbf{s}_{\text {new }}\right), \ldots, k\left(\mathbf{s}\left(N_{T}\right), \mathbf{s}_{\text {new }}\right)\right]$.

Assuming that the variance $\sigma^{2}$ represents the noise distribution, the prior distribution can be expressed as follows:

$\mathbf{d}^{\prime} \sim N\left(0,\left[\begin{array}{cc}\mathbf{K} & \mathbf{k} \\ \mathbf{k}^{\top} & k\left(\mathbf{s}_{\text {new }}, \mathbf{s}_{\text {new }}\right)\end{array}\right]+\sigma^{2} \mathbf{I}\right)$, where $\mathbf{d}^{\prime}=\left[d(1), \ldots, d(N t), d_{n e w}\right]^{\top}$. Since $\mathbf{d}$ is known, the posterior distribution $p\left(d_{n e w} \mid \mathbf{d}\right)$ can be obtained. The expected value of the posterior distribution is then used as the predicted value, and we denote it as $\hat{d}_{\mathrm{GP}}(n)$ :

$\hat{d}_{\mathrm{GP}}(n)=\mathbf{k}^{\top}\left(\mathbf{K}+\sigma^{2} \mathbf{I}\right)^{-1} \mathbf{d}$.

As shown in Eq. (4), the maximum tsunami height of the test data is estimated by the weighted sum of the kernel function of all training data. The terms $\beta$ and $\sigma^{2}$ shown above are hyperparameters, which we optimized these hyper-parameters using cross-validation and grid-search approaches.

However, while GP regression is useful as a powerful nonlinear multivariate interpolation tool (Rasmussen and Williams 2006), its prediction accuracy cannot be guaranteed for inputs with sparse training data. Therefore, GP regression does not perform extrapolation well, although extrapolation is fundamental for predicting beyond the range of training data. To solve this problem, we must know the relationship among the data and introduce that relationship into a prediction model.

\section{Nonlinear parametric model for estimating the tsunami height}

In this study, we propose a nonlinear parametric model to predict the coastal tsunami height from pressure gauge data by using a power law relationship between the tsunami height and gauge data as shown in Fig. 4b. Figure 4b shows that the relationship between the water pressure gauge data and the maximum tsunami height on a log-log graph and appears to be linearly distributed, and thus, this relationship is called a power law.

We mathematically express the power law relationship as follows. 
$d=a s^{b}$,

where $a$ and $b$ are constant numbers. Upon taking the logarithm of both sides in Eq. (5), we can write the power law relationship as $\log d=b \log s+\log a$. We can interpret $b$ as the inclination and $\log a$ as the intercept in the $\log -\log$ graph.

We extend Eq. (5) to constitute a multivariate relationship, and then estimate $\hat{d}_{\mathrm{NP}}$ by the nonlinear parametric model can be obtained as follows:

$\hat{d}_{\mathrm{NP}}(n)=\sum_{i=1}^{S} a_{i} s_{i}(n)^{b_{i}}$,

where the parameters are $a_{i}$ and $b_{i}$ are optimized so that the mean square error is minimized. However, it is considered difficult to optimize these parameters analytically because of the model complexity; therefore, we use a gradient descent method to achieve optimization. In the gradient descent method, the parameters are initially set to certain values, and optimization is performed by updating the parameters so that the errors decrease.

We formulate the gradient descent method as follows. We establish a loss function as the mean squared error according to, $E\left(a_{i}, b_{i}\right)=\frac{1}{n} \sum_{n}\left|d_{\mathrm{NP}}(n)-d(n)\right|^{2}$. Then, the update formulas are written as follows:

$a_{i} \leftarrow a_{i}-\epsilon \frac{\partial}{\partial a_{i}} E\left(a_{i}, b_{i}\right), b_{i} \leftarrow b_{i}-\epsilon \frac{\partial}{\partial b_{i}} E\left(a_{i}, b_{i}\right)$,

where $\epsilon$, which is the named learning coefficient, is set as a parameter of the gradient descent method. If we use sufficiently small values of $\epsilon$ in the above update formulas, the loss function certainly decreases. In this study, we initialized $a_{i}=0, b_{i}=1$, and we set $\epsilon=0.01$.

\section{Results}

In this section, we investigate the prediction performance using three methods: the MM regression approach, the GP regression technique, and the nonlinear parametric model. In "Regression" section, we perform regression for a training data set to investigate whether each methodology can fit the training data set. We then investigate the prediction performance of each methodology using test data in both "Prediction performance under expected circumstances" and "Prediction performance under unexpected circumstances" sections, in which we investigate the prediction performance of each method under both expected circumstances and unexpected circumstances, respectively.

\section{Regression}

We first investigate the ability of the proposed nonlinear parametric model to predict the tsunami height from the water pressure gauge data and compare our proposed method with the two previous methods (Baba et al. 2014; Igarashi et al. 2016). First, we perform regression for the tsunami height using the maximum value of the average absolute value observed by the water pressure gauge $s(n)$. This process is a one-dimensional regression approach, and thus, the result is easy to visualize.

The one-dimensional regression results are shown in Fig. 5a, where the gray points represent the training data, and the colored lines signify the regression line of each method. The tsunami height predicted using MM regression tends to be bigger than the actual tsunami height when the observed value is high and tends to be smaller than the actual tsunami height when the observed value is low. On
Fig. 5 One-dimensional regression results of the relationship between $s(n)$ and $d(n)$. Gray points represent training data, while white points show intentionally removed data. This figure shows the regression lines: the blue line shows the MM regression result, the green line shows the GP regression result; the red line shows the result of our proposed method

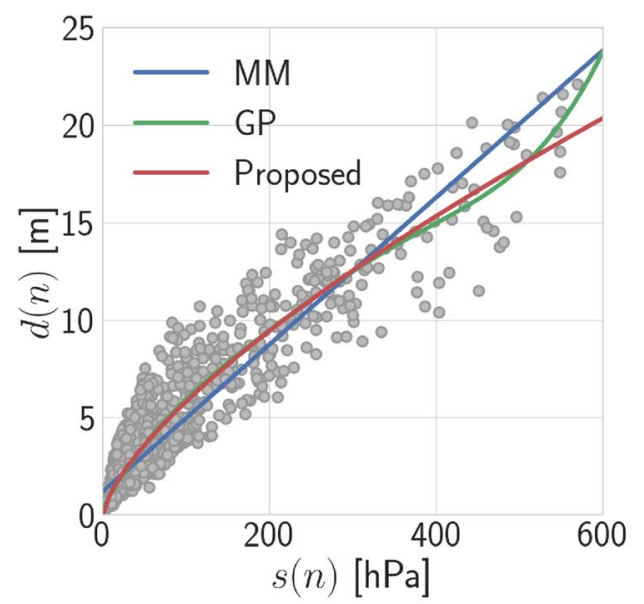

(a) Using all data

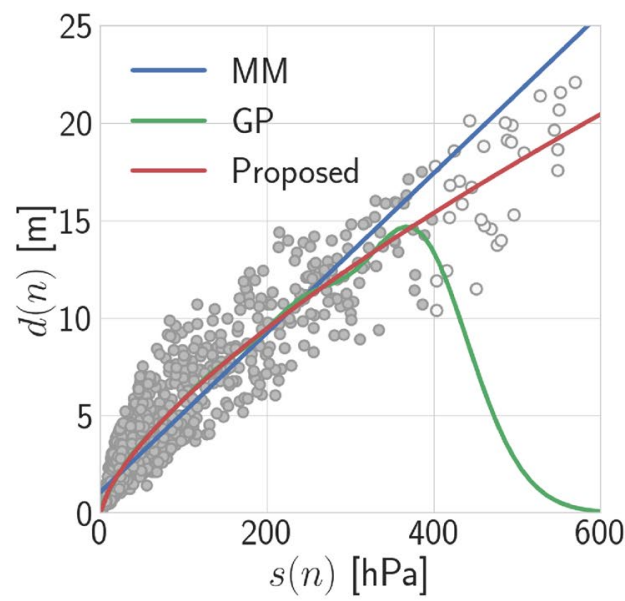

(b) Intentionally removing data with $s(n) \leq 400$ 
the other hand, GP regression and our proposed method both predict the tsunami height with relatively less bias.

To investigate whether each methodology can fit the training data set, we calculated an estimation error using the root mean squared error (RMSE) as follows:

RMSE $=\sqrt{\frac{1}{N_{D}} \sum_{n \in D}\{d(n)-\hat{d}(n)\}^{2}}$,

where $D$ is the dataset utilized to calculate the RMSE, and $N_{D}$ is the size of the dataset. Then, we compared the accuracy of each method quantitatively. In the one-dimensional regression, the RMSE values generated by the MM and GP regression techniques and our proposed method are $1.31 \mathrm{~m}$, $1.16 \mathrm{~m}$, and $1.16 \mathrm{~m}$, respectively. Thus, GP regression and our proposed method can predict the tsunami height with a better accuracy than can MM regression and can effectively learn the nonlinear relationship between ocean pressure gauge data and coastal tsunami heights.

Using test data to calculate the prediction error represented by the RMSE in Eq. (8), we can verify the prediction performance of each method. Especially with regard to the one-dimensional case, we focus on the prediction performance under unexpected circumstances to predict the tsunami height with inputs far from training data; this approach mimics the so-called extrapolation problem in machine learning. Unlike the previous results, it is difficult for GP regression to predict the tsunami height under unexpected circumstances (Rasmussen and Williams 2006). To demonstrate the extrapolation problem with GP regression, we perform one-dimensional regression with training data of $s \leq 400$ only (i.e., we intentionally remove the data with $s>400$ ). The data with $s \leq 400$ are plotted in Fig. $5 \mathrm{~b}$ as gray points, and all other as white points.

While GP regression makes predictions with relatively little bias in the range of the training data set, the prediction error far exceeds the range of the training data, as shown by the green line in Fig. $5 \mathrm{~b}$. In fact, the estimation error and a prediction error using GP regression under unexpected circumstances for the gray points and white points are $1.11 \mathrm{~m}$ and $12.28 \mathrm{~m}$, respectively. In contrast, our proposed method makes predictions with little bias even when the training data does not exist, as shown by the red line in Fig. 5b. The errors generated by our proposed method for the gray points and white points are $1.12 \mathrm{~m}$ and $3.35 \mathrm{~m}$, respectively. Evidently, our proposed method makes predictions with fewer errors than GP regression when we estimate the tsunami height from DONET observations through extrapolation.

Thus far, we have performed one-dimensional regression to illustrate the advantages of our proposed method with regard to the extrapolation problem. Next, we extend the GP regression technique and our proposed method are easily extended to implement multidimensional regression, which is required for practical matters. We perform multidimensional regression using a randomly selected training data set (1004 cases), and Fig. 6a shows the multidimensional regression fitting results. The colored points in Fig. 6a show the values predicted by each method; the horizontal axis denotes the actual tsunami height, while the vertical axis represents the predicted tsunami height, and the gray line shows the $d=\hat{d}$ line. The predicted points are closer to the $d=\hat{d}$ line in the presence of fewer prediction errors.

As with one-dimensional regression, the tsunami height predicted by MM regression tends to be bigger when the observed value is high, and vice versa. However, GP regression and our proposed method predict the tsunami height with relatively less bias. The RMSE values generated by the MM and GP regression techniques and by our proposed method are $1.32 \mathrm{~m}, 0.55 \mathrm{~m}$, and $0.76 \mathrm{~m}$, respectively. We performed the same regression 100 times while changing the seed of the random number generator to avoid accidental results caused by randomly choosing training data. Figure $6 \mathrm{c}$ shows the average of fitting errors and error bars, which signify mean a $95 \%$ confidence interval. The averaged RMSE values generated by the MM and GP regression techniques and by our proposed method are $1.31 \mathrm{~m}, 0.57 \mathrm{~m}$, and $0.76 \mathrm{~m}$, respectively. GP regression makes predictions with fewer errors than our proposed method; despite this, our proposed method improves the prediction accuracy compared with MM regression.

It is difficult to know which DONET observation point is important for the prediction by interpreting the GP regression results because GP regression is a nonparametric method, and thus, we cannot obtain the parameters from GP regression. In our proposed method, which is a parametric method, we can obtain the parameters by regression and therefore interpret the results to ascertain the importance of each observation point for the prediction. In Fig. 7 we show the parameters obtained during the experiment illustrated in Fig. 6a. Some values of the weight parameters, $a_{i}$, are closer to zero, whereas others are larger in the positive direction or the negative direction. The parameters of the power law $b_{i}$ are all positive. In the range of $i$ in which $a_{i}$ is small, a change in the DONET observation value $s_{i}(n)$ effects the prediction value $\hat{d}_{\mathrm{NP}}(n)$ only slightly.

\section{Prediction performance under expected circumstances}

Thus far, we have used given data to compare the regression performance of the proposed method with those of the previous methods. Next, we use test data to verify the prediction performance of each method. First, we assumed that the test 
Fig. 6 Results of the prediction experiment. a Relationship between the simulated tsunami height $d(n)$ and the tsunami height $\hat{d}(n)$ estimated from the training data set. Blue points show the estimation results of MM regression, green points show the estimation results of GP regression, and red points show the estimation results of our proposed method. $\mathbf{b}$ Relationship between the simulated tsunami height $d(n)$ and the tsunami height $\hat{d}(n)$ predicted from the test data set. c Comparison of the RMSE estimation errors for training set with two-sided $95 \%$ confidence intervals. d Comparison of the RMSE prediction errors for the test dataset with two-sided 95\% confidence intervals

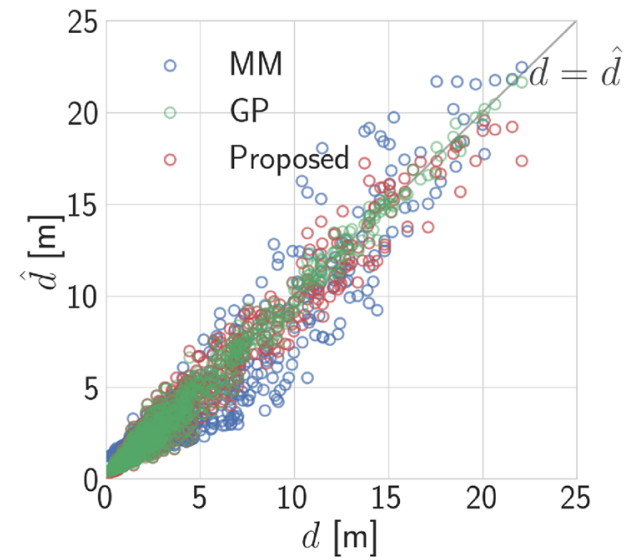

(a)

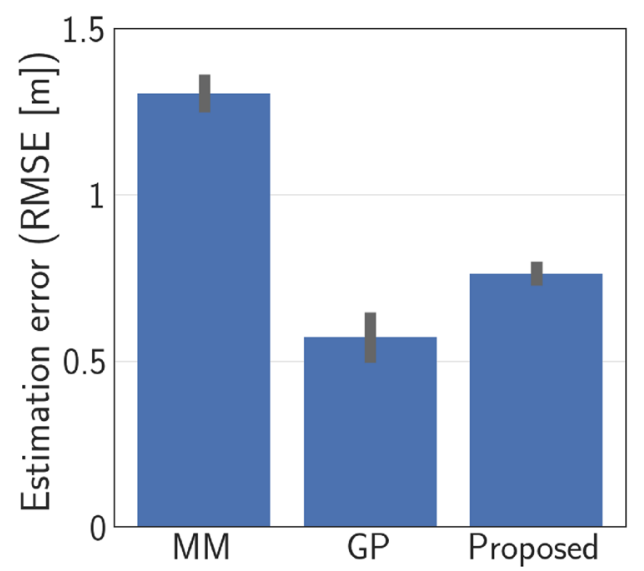

(c)

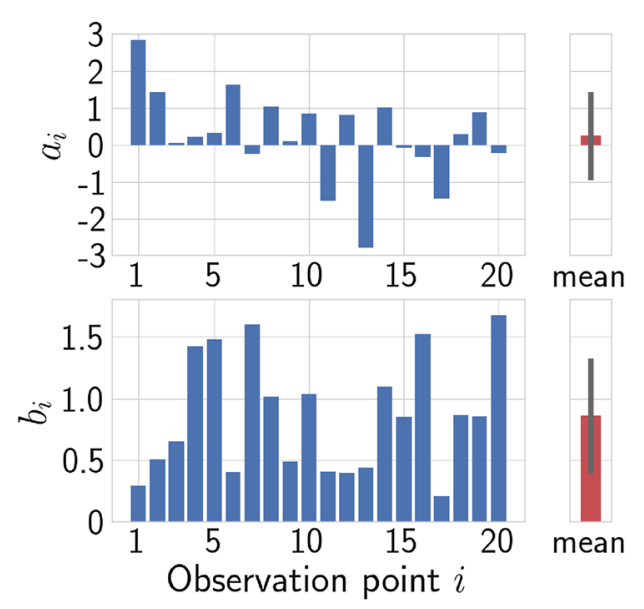

(a)

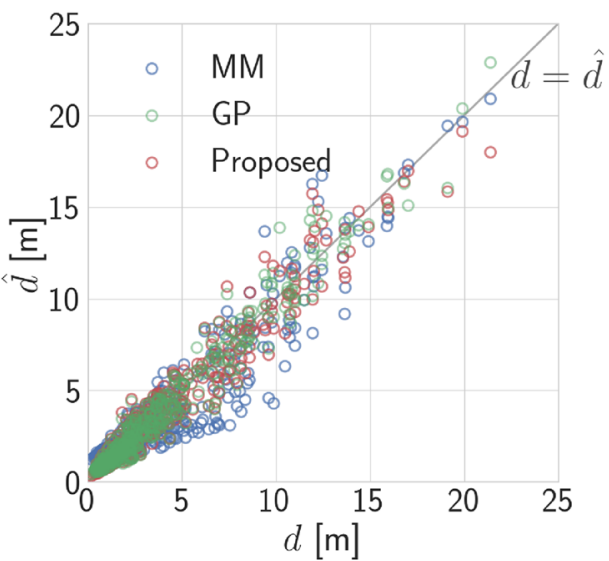

(b)

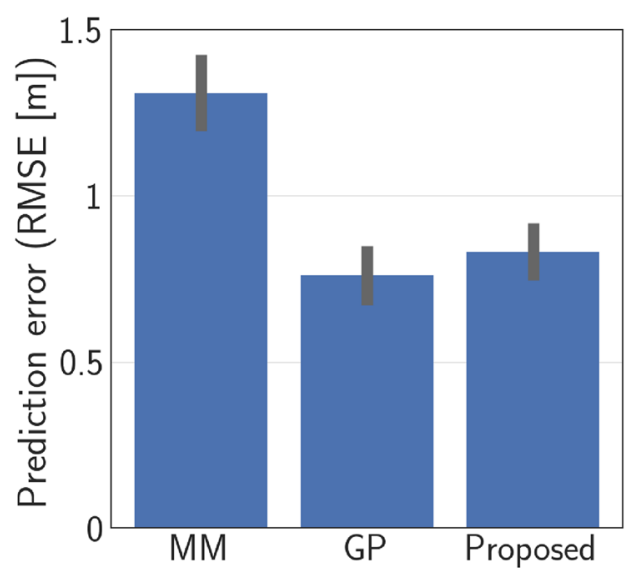

(d)

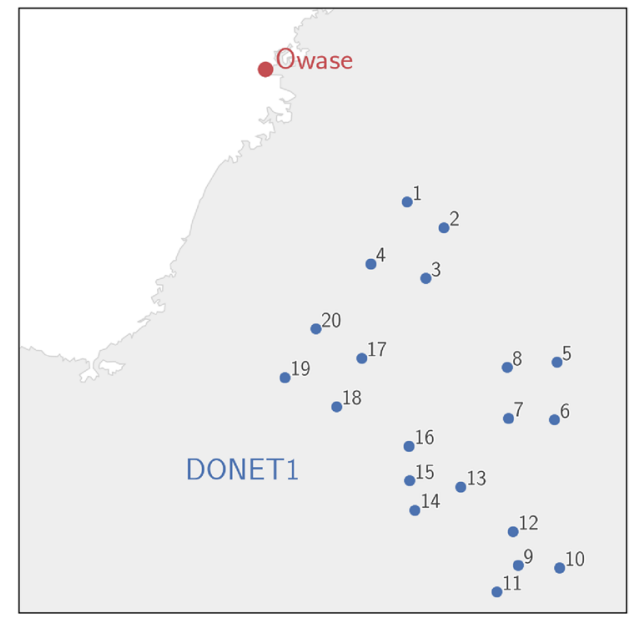

(b) data are drawn from the same population as the training data, and we investigated the prediction performance of each method under expected circumstances. Following a previous study (Igarashi et al. 2016), we randomly shuffled all data points (1506 cases) and then divided them into the training set (1004 cases) for learning and the test set (502 cases) for verifying the prediction accuracy as shown in Fig. 8a. We then interpret the test data as unknown data under expected 
Fig. 8 Dataset used in the prediction experiments. Black points represents the training data set, and the white points represents the test data set. a Randomly selected data set used in the experiment illustrated in Fig. 6. b Data set used to verify the extrapolation accuracy

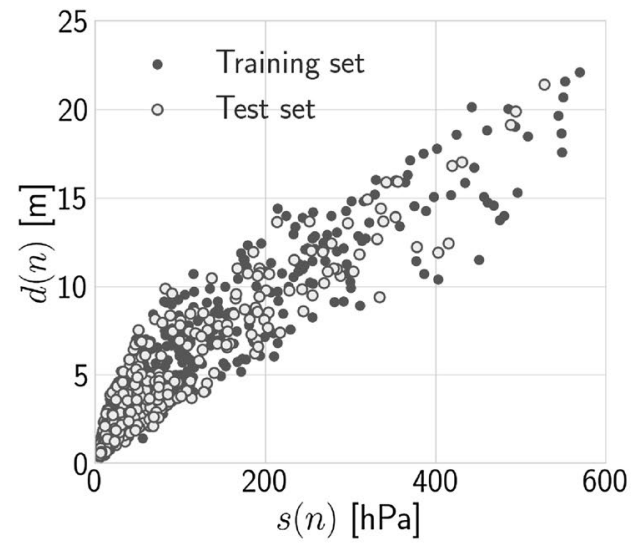

(a)

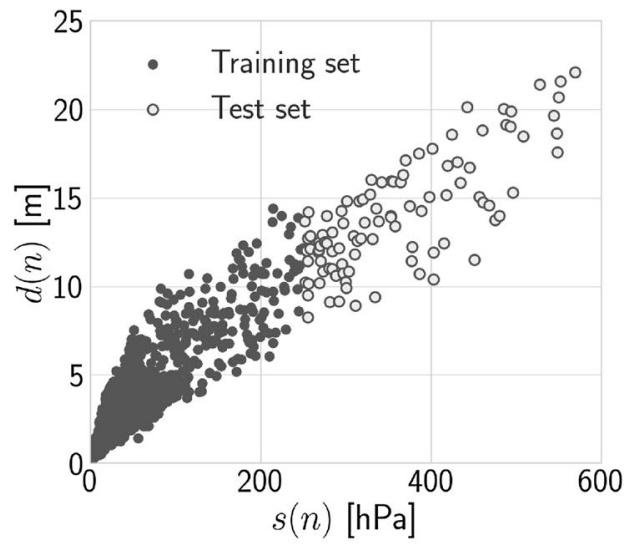

(b) circumstances. Figure $6 \mathrm{~b}$ shows the prediction performance of each method under expected circumstances. The gray line represents the $d=\hat{d}$ line, to which the predicted points are closer in the presence of fewer prediction errors. The values predicted by MM regression tend to be smaller when $5 \leq d \leq 10$; furthermore, MM regression generates bias, as shown in Fig. 6b. In contrast, GP regression and our proposed method appear to predict the tsunami height with relatively less bias. The RMSE values generated by MM and GP regression and by our proposed method are $1.28 \mathrm{~m}, 0.74 \mathrm{~m}$, and $0.81 \mathrm{~m}$, respectively; the RMSE values generated by our proposed method are smaller than those of MM regression by $36 \%$ and larger than those of GP regression by $9 \%$. This finding indicates that our proposed method can predict the coastal height of a tsunami with approximately the same accuracy as can GP regression.

We performed the same prediction experiments 100 times for each method while changing the seed of the random number generator to avoid accidental results caused by randomly choosing the training data. Figure $6 \mathrm{~d}$ shows the average of each prediction error and error bars, which signify a 95\% confidence interval. Averaged RMSE by MM, GP, and our proposed method are $1.31 \mathrm{~m}, 0.76 \mathrm{~m}$, and $0.83 \mathrm{~m}$, respectively. Averaged RMSE by our proposed method is smaller than that of MM by $37 \%$ and larger than that of GP by $9 \%$. This also indicates that our proposed method can predict the coastal tsunami height with approximately the same accuracy as can GP regression.

\section{Prediction performance under unexpected circumstances}

In the previous section, we assume that the test data are drawn from the same population as the training data, and we investigate the prediction performance of each method under expected circumstances. However, in practice, we cannot deny the possibility that the coastal tsunami height can exceed the scope of the assumption. Thus, in this subsection, we investigate the prediction error under a scenario of unexpected circumstances, which is often called extrapolation, using the three methods, namely, the MM and GP regression techniques and our proposed nonlinear parametric model. To verify the prediction performance of each method under unexpected circumstances, we sorted all data (1506 cases) by the water pressure gauge observation value $s(n)$ and then divide them into the training set (1406 cases) for learning and the test set (100 cases) for verification, as shown in Fig. $8 \mathrm{~b}$. Using the test data set, we evaluated the prediction performance of each method under unexpected circumstances, that is, when the coastal tsunami height exceeds the scope of the assumption.

To clearly show the prediction performance of each method under unexpected circumstances, we perform onedimensional regression with the abovementioned training data set (1406 cases) and evaluate the test data set (100 cases). In the one-dimensional regression scenario, we use the MM, $s(n)=\max _{t} \frac{1}{S} \sum_{i=1}^{S}\left|p_{i}(n, t)\right|$, as an input variable, where $n$ denotes scenario $n$ of the training set. Similar to Figs. 5b, 9a shows that our proposed method makes predictions with relatively little bias even the training data do not exist, whereas the prediction error of GP regression largely exceeds the range of training data. The predictions values of GP regression in $s(n)>250$ are directly affected by the rightmost training data, and they exponentially increase as a result, as shown in Fig. 9a. In fact, calculating the RMSE values generated by GP regression and our proposed method for the white points to evaluate the prediction performance of both methods under unexpected circumstances, we found that the GP regression and MM regression techniques and proposed method generated RMSE values of 165.7, 6.2 and $4.1 \mathrm{~m}$, respectively.

Thus far, we have performed one-dimensional regression to illustrate the advantages of our proposed method. Next, let us consider multidimensional regression, using 


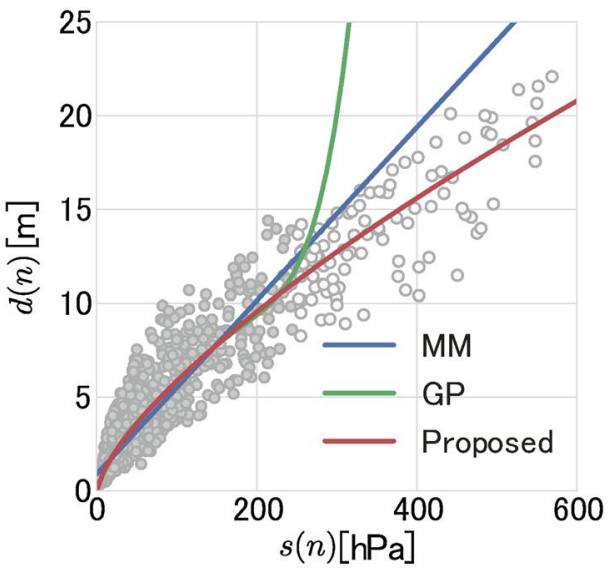

(a)

Fig. 9 Extrapolation prediction results for the test set in Fig. 8b. a One-dimensional data set used to verify the extrapolation accuracy and regression results for extrapolation prediction. This figure shows the regression lines: the blue line shows the MM regression result, the green line shows the GP regression result, the red line shows the

$\mathbf{s}(n)=\left[s_{1}(n), \ldots, s_{S}(n)\right]$ as the input variables. Similar to the results of one-dimensional regression results, Fig. $9 \mathrm{~b}$ shows that, MM regression and our proposed method provide relatively accurate predictions, whereas GP regression fails to predict the tsunami height due to a deficit of water pressure gauge data. In fact, the MM regression method, GP regression technique, and our proposed method generated RMSE values of $4.30 \mathrm{~m}, 10.72 \mathrm{~m}$, and $3.16 \mathrm{~m}$, respectively; the RMSE generated by our proposed method is smaller than that generated by MM regression by $27 \%$ and smaller than that generated by GP regression by $71 \%$.

The differences in the prediction performance of the methods under unexpected circumstances are due to whether the prediction methods are parametric or nonparametric models. Regression models are classified as either parametric or nonparametric models. While GP regression is classified as a nonparametric model, MM regression and our proposed method are both parametric models, in which the relationship between the data can be modeled by expressing the relationship through a parameterized formula. Although strong assumptions are not placed on the relationship in nonparametric models and flexible modeling can be performed, the regression results are strongly affected by the data; furthermore, nonparametric models cannot offer effective predictions beyond the range of training data, and thus, they exhibit a low extrapolation accuracy. Theoretically, Eq. (4), which gives us the theoretical results of GP regression, shows that the Gaussian kernel between the test data and training data, $\mathbf{k}$, drops sharply to zero in the case of a prediction beyond the range of training data well, and the predicted tsunami height becomes 0, as shown in Fig. 9b. Although

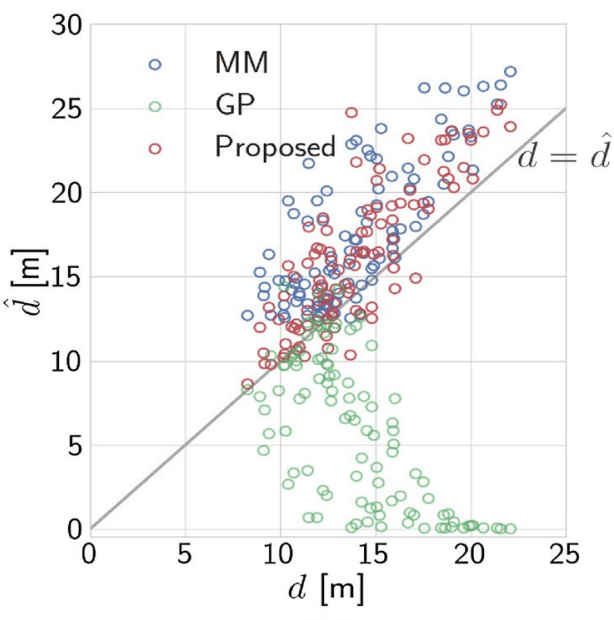

(b)

result of our proposed method. b Multidimensional regression results for extrapolation prediction. This figure shows the regression results: the blue points show the MM regression result, the green points show the GP regression result, the red points show the result of our proposed method

Fig. 9a shows that the predictions values of GP regression in $s(n)>250$ are directly affected by the rightmost training data and thus exponentially increased, $s(n)$ increases, and the predictive tsunami height also becomes 0 . Therefore, our proposed method and MM regression are much better than GP regression at predicting the tsunami height under unexpected circumstances.

\section{Discussion}

In this study, we find that there is a power law relationship exists between the water pressure gauge data and the tsunami height and accordingly construct a nonlinear parametric model on the basis of this power law relationship. However, in practice, we cannot deny the possibility that the coastal tsunami height will exceed the scope of the assumption; therefore, we evaluate the prediction performance of the three methods under a scenario of unexpected circumstances, which is called extrapolation. Following a verification, we found that our proposed method increases the prediction performance under the extrapolation scenario in comparison with GP regression. In this study, we verify the ability of our proposed method to extrapolate data with higher water pressures. However, as another case of extrapolation, the ability of our proposed method to extrapolate for nonuniform fault models is considered. In addition, for further verification, simulating tsunami scenarios are simulated with nonuniform fault models, and performing prediction experiments are necessary. 
Let us discuss the applications of our method to real data. In practice, physical phenomena such as seismic and ocean acoustic waves are abundant; nevertheless, our simulation does not take these phenomena into account. However, because the periods of tsunami waves are much higher than those of these physical phenomena, a preprocessing system using low-pass filtering can work effectively at removing these phenomena (Takahashi et al. 2017). Thus, the application of our proposed model into real data is considered effective, and the application of our method to the real data must be further investigated.

From the perspective of machine learning, there are a few problems in our proposed method. First, when our proposed method is trained using training data, there is a possibility that the model will be affected by the noise included within the training data. This problem is known as overfitting, and MM regression and GP regression also exhibit this problem. Second, our proposed method is a somewhat complicated model; consequently, local minima may exist, and thus, it is necessary to verify whether local minima actually exist and to address them accordingly. Finally, in this study, to enhance the prediction performance, the prediction model does not take a physical model into account and the coefficient of the prediction model can be either negative or positive. As a result, some of the coefficients $a_{i}$ are negative, as shown in Fig. 7, which may does not make physical sense. Thus, further investigation is needed to construct a model for predicting the height of a tsunami under the constraint of positive coefficients and to study the relationship between this phenomenological model and a physical tsunami model.

Let us consider the limitation of our proposed method insomuch that it depends on the study area. In this study, we choose Osawa in Kii Prefecture, Japan, as the target region because Owase represents the front of the DONET ocean bottom pressure gauges and because there is a strong positive correlation between the maximum tsunami height at Owase and the maximum value of the absolute water pressure (Baba et al. 2014). We thus assume the existence of a positive correlation between the ocean bottom gauges and tsunami height in the prediction region, but this assumption restricts the application of our method to other regions. To apply our method to other regions, we have to select appropriate gauges for the tsunami prediction using machine learning methods. In our previous study (Taniguchi et al. 2018), we used the prior knowledge that the important points for tsunami height prediction is sparse and show the sparse modeling (Tibshirani 1996; Bishop 2006) is useful for automatic selection of ocean bottom gauges. Therefore, further investigation is needed to combine the sparse modeling and our proposed method for overcoming the limitation of our proposed method depending on the study area.

Next, let us discuss the case in which our proposed method has a substantial advantage over the other two methods. Based on the strong positive correlation between the observed water pressure data and the costal tsunami height in Owase, a previous study (Baba et al. 2014) assumed a linear relationship with the MM regression method for the sake of simplicity. In contrast, our proposed method uses a nonlinear power law relationship, which automatically expresses the strong positive correlation in Owase. Thus, when this nonlinear relationship is preserved in the prediction dataset, the tsunami prediction performance of our proposed method is better than that of the MM regression method, even under unexpected circumstances. Moreover, although the prediction method using GP regression does not assume the existence of this relationship, the GP regression method cannot offer predictions exceeding the range of training data well, and its prediction performance under unexpected circumstances is worse than those of the other two methods.

As shown in a previous study (Baba et al. 2014), the abovementioned nonlinear relationship is preserved in other areas in the Kii Peninsula such as Kumano and Uragami in Kii Peninsula, which are the fronts of the DONET ocean bottom pressure gauges. In the future work, it will be necessary to investigate the effects of the local bathymetry (due to wave shoaling) on the power law relationship using massive scenarios of tsunami simulation in other regions simultaneously with the selection of ocean pressure gauges for tsunami prediction. Moreover, further investigation is needed to explain how this phenomenological nonlinear relationship relates to a physical model, which would helps us to understand tsunamis in a broader context and implement rapid tsunami prediction schemes in various coastal regions.

\section{Conclusion}

In this study, we found the existence of a power law relationship between water pressure gauge data and the tsunami height, and we constructed a nonlinear parametric model to predict the tsunami height. We performed prediction experiments to verify the accuracy of our proposed method, the results of which indicate that our proposed method can offer predictions with approximately the same accuracy as GP regression. However, in practice, we cannot deny the possibility that the coastal tsunami height will exceed the scope of the assumption; therefore, we evaluated the prediction performance under unexpected circumstances, which is called extrapolation. As a result, we found that the extrapolation accuracy of our proposed method is higher than those of MM regression and GP regression.

The results reported in this paper are based on a nonlinear power law relationship, which authentically expresses the strong positive correlation in Owase. Therefore, to overcome the limitation of our proposed method insomuch that 
it depends on the study area, further investigation is needed to understand the effects of the local bathymetry (due to wave shoaling) on the relationship using massive scenarios of tsunami simulations in other regions simultaneous with the selection of ocean pressure gauges for tsunami prediction. Moreover, further investigation is needed to explain how this phenomenological nonlinear relationship relates to a physical model, which would helps us to understand tsunamis in a broader context and implement rapid tsunami prediction schemes in various coastal regions.

Acknowledgements Part of this research was financially supported by a Grant-in-Aid for Scientific Research on Innovative Areas (No. 25120009) and Grant-in-Aid for Young Scientists (B) (No. 17K12735) from the Ministry of Education, Culture, Sports, Science and Technology of Japan, and CREST Project establishing the most advanced disaster reduction management system by fusion of real-time disaster simulation and big data assimilation (No. JPMJCR1411) from the Japan Science and Technology Agency.

Open Access This article is distributed under the terms of the Creative Commons Attribution 4.0 International License (http://creativeco mmons.org/licenses/by/4.0/), which permits unrestricted use, distribution, and reproduction in any medium, provided you give appropriate credit to the original author(s) and the source, provide a link to the Creative Commons license, and indicate if changes were made.

\section{References}

Baba T, Cummins PR (2005) Contiguous rupture areas of two Nankai Trough Earthquakes revealed by high-resolution tsunami waveform inversion. Geophys Res Lett. https://doi.org/10.1029/2004G L022320

Baba T, Tanioka Y, Cummins PR, Uhira K (2002) The slip distribution of the 1946 Nankai Earthquake estimated from tsunami inversion using a new plate model. Phys Earth Planet Inter 132(1):59-73

Baba T, Hirata K, Kaneda Y (2004) Tsunami magnitudes determined from ocean-bottom pressure gauge data around Japan. Geophys Res Lett. https://doi.org/10.1029/2003GL019397

Baba T, Takahashi N, Kaneda Y (2014) Near-field tsunami amplification factors in the Kii Peninsula, Japan for Dense Oceanfloor Network for Earthquakes and Tsunamis (DONET). Mar Geophys Res 35(3):319-325

Baba T, Ando K, Matsuoka D, Hyodo M, Hori T, Takahashi N, Obayashi R, Imato Y, Kitamura D, Uehara H et al (2016)
Large-scale, high-speed tsunami prediction for the Great Nankai Trough Earthquake on the K computer. Int J High Perform Comput Appl 30(1):71-84

Bishop CM (2006) Pattern recognition and machine learning (information science and statistics). Springer, Berlin

Hayashi Y (2010) Empirical relationship of tsunami height between offshore and coastal stations. Earth Planets Space 62(3):269-275

Igarashi Y, Hori T, Murata S, Sato K, Baba T, Okada M (2016) Maximum tsunami height prediction using pressure gauge data by a Gaussian process at Owase in the Kii Peninsula, Japan. Mar Geophys Res 37(4):361-370

Kanamori H (1972) Tectonic implications of the 1944 Tonankai and the 1946 Nankaido Earthquakes. Phys Earth Planet Inter 5:129-139

Kaneda Y, Kawaguchi K, Araki E, Matsumoto H, Nakamura T, Kamiya S, Ariyoshi K, Hori T, Baba T, Takahashi N (2015) Development and application of an advanced ocean floor network system for megathrust earthquakes and tsunamis. In: Seafloor observatories. Springer, Berlin, pp 643-662

Kocijan J, Murray-Smith R, Rasmussen CE, Girard A (2004) Gaussian process model based predictive control. In: Proceedings of the 2004 American control conference, 2004, vol 3. IEEE, pp 2214-2219

Krause A, Singh A, Guestrin C (2008) Near-optimal sensor placements in Gaussian processes: theory, efficient algorithms and empirical studies. J Mach Learn Res 9(Feb):235-284

Rasmussen CE, Williams CK (2006) Gaussian processes for machine learning, vol 1. MIT Press, Cambridge

Takahashi N, Imai K, Ishibashi M, Sueki K, Obayashi R, Tanabe T, Tamazawa F, Baba T, Kaneda Y (2017) Real-time tsunami prediction system using DONET. J Disaster Res 12(4):766-774

Taniguchi J, Tagawa K, Yoshikawa M, Igarashi Y, Ohsumi T, Fujiwara H, Hori T, Okada M, Baba T (2018) Selection of tsunami observation points suitable for database-driven prediction. J Disaster Res 13(2):245-253

Tibshirani R (1996) Regression shrinkage and selection via the Lasso. J R Stat Soc B 58:267-288

Tsushima H, Hino R, Fujimoto H, Tanioka Y, Imamura F (2009) Nearfield tsunami forecasting from cabled ocean bottom pressure data. J Geophys Res Solid Earth. https://doi.org/10.1029/2008JB0059 88

Tsushima H, Hirata K, Hayashi Y, Tanioka Y, Kimura K, Sakai S, Shinohara M, Kanazawa T, Hino R, Maeda K (2011) Near-field tsunami forecasting using offshore tsunami data from the 2011 off the Pacific Coast of Tohoku Earthquake. Earth Planets Space 63(7):56

Utsu T (2001) Seismology. Kyoritsu Publishers, Tokyo 